\title{
Rose Window Graphs
}

\author{
Steve Wilson \\ Department of Mathematics and Statistics \\ Northern Arizona University, Flagstaff, AZ 86011, USA
}

Received 25 July 2007, accepted 17 May 2008, published online 17 June 2008

\begin{abstract}
This paper introduces a family of tetravalent graphs called rose window graphs, denoted by $R_{n}(a, r)$, and investigates their symmetry properties. Four families of these graphs are shown to be edge-transitive and it is conjectured that every $R_{n}(a, r)$ which is edge-transitive belongs to one of these families. Proofs and conjectures about the size of a dart-stabilizer and about regular maps containing these graphs are also offered.
\end{abstract}

Keywords: Graph, automorphism group, symmetry, edge-transitive graph, regular map, tetravalent graph, rose window.

Math. Subj. Class.: 05C25, 20F65

\section{Introduction}

The graphs considered in this paper arose from a talk by the author at GEMS ' 97 concerning regular maps and their underlying graphs; the talk later became the paper [5]. At the end, it shows pictures of five graphs of maps, the smallest graphs not then known to be part of a family of graphs. As reported in [5], after the talk, Dragan Marušič and Tomaž Pisanski observed that one of the graphs could be redrawn to give three of the graphs very similar figures. The generalization of those three pictures is the topic of this paper.

\section{Definitions}

In this paper, all graphs considered are simple graphs. The graph $\Gamma$ has vertex set $\mathrm{V}(\Gamma)$ and edge set $\mathrm{E}(\Gamma)$. A symmetry or automorphism of a graph is a permutation of $\mathrm{V}(\Gamma)$ which preserves $\mathrm{E}(\Gamma)$. The symmetries of $\Gamma$ form a group, $\operatorname{Aut}(\Gamma)$, under composition. Each edge $\{u, v\}$ of $\Gamma$ corresponds to two directed edges, also called darts or arcs; one is $(u, v)$, the other is $(v, u)$.

E-mail address: stephen.wilson@nau.edu (Steve Wilson) 
A map $\mathcal{M}$ is an embedding of a graph $\Gamma$ in a surface $S$ so that the faces, i.e., the connected components of the complement of the graph, are simply-connected. A symmetry or automorphism of a map $\mathcal{M}$ is a homeomorphism of $S$ onto itself which preserves $\mathcal{M}$. Alternately, a symmetry of $\mathcal{M}$ is a symmetry of $\Gamma$ which preserves the collection of cycles of edges which surround faces of $\mathcal{M}$. The map $\mathcal{M}$ is rotary provided that $\operatorname{Aut}(\mathcal{M})$ contains symmetries $R$ and $S$ which act on some incident face-vertex pair (these are the central face and vertex) as rotations one step about the face and the vertex, respectively. Then $\gamma=R S^{-1}$ acts as a $180^{\circ}$ flip about the center of an edge, and thus has order 2. A rotary map $\mathcal{M}$ is reflexible provided that $\operatorname{Aut}(\mathcal{M})$ also contains symmetries which act locally as reflections. A map which is rotary but not reflexible is chiral. If $\mathcal{M}$ is rotary, its group of symmetries acts transitively on the darts of $\Gamma$. In the literature, the word "regular" is also used, sometimes with the meaning of "rotary", sometimes with the meaning of "reflexible".

In a rotary map $\mathcal{M}, \operatorname{Aut}(\mathcal{M})$ acts transitively on faces and on vertices. Thus, all faces are $p$-cycles for some $p$, and all vertices are $q$-valent for some $q$. We then say the map is of type $\{p, q\}$.

A Petrie path in a map is a cycle of edges in which each two consecutive belong to the same face, but no three consecutive do. The map $\mathrm{P}(\mathcal{M})$ is formed from $\mathcal{M}$ by dissolving every face, and then identifying each Petrie path with the boundary of a topological disk. Clearly, $\mathcal{M}$ and $\mathrm{P}(\mathcal{M})$ have the same underlying graph. If $\mathcal{M}$ is reflexible, so is $\mathrm{P}(\mathcal{M})$, while if $\mathcal{M}$ is chiral, $\mathrm{P}(\mathcal{M})$ is not rotary.

If $\mathcal{M}$ is reflexible, all its Petrie paths have the same length $r$. We say $\mathcal{M}$ is of type $\{p, q\}_{r}$, and then $\mathrm{P}(\mathcal{M})$ is of type $\{r, q\}_{p}$.

See [5] for a more thorough description of these notions.

Definition 1. The Rose Window graph $R_{n}(a, r)$ has $2 n$ vertices: $A_{i}, B_{i}$ for $i \in \mathbb{Z}_{n}$; all arithmetic with $r, a$ and vertex subscripts is assumed to be done in $\mathbb{Z}_{n}$. The graph has four kinds of edges:

Rim: $\quad A_{i}-A_{i+1}$

In-Spoke: $\quad A_{i}-B_{i}$

Out-spoke: $\quad B_{i}-A_{i+a}$

Hub: $\quad B_{i}-B_{i+r}$

For example, Figure 1 shows $R_{10}(2,3)$.

In this and other figures in this paper, vertices labeled $1 \ldots n$ are vertices $A_{1}, A_{2}, \ldots, A_{n}$, while those labeled $n+1, n+2, \ldots, 2 n$ are $B_{1}, B_{2}, \ldots, B_{n}$.

Some facts about these graphs can be seen at once. First

$$
R_{n}(a, r) \cong R_{n}(-a, r)
$$

An isomorphism is given by $A_{i} \rightarrow A_{-i}, B_{i} \rightarrow B_{-i}$. Also,

$$
R_{n}(a, r)=R_{n}(a,-r)
$$

Notice the equal sign; these graphs are identical, not just isomorphic. Also, if $(n, r)=1$, then

$$
R_{n}(a, r) \cong R_{n}\left(a r^{-1}, r^{-1}\right),
$$

the isomorphism being given by $A_{i} \rightarrow B_{-i r^{-1}}, B_{i} \rightarrow A_{-i r^{-1}}$. 


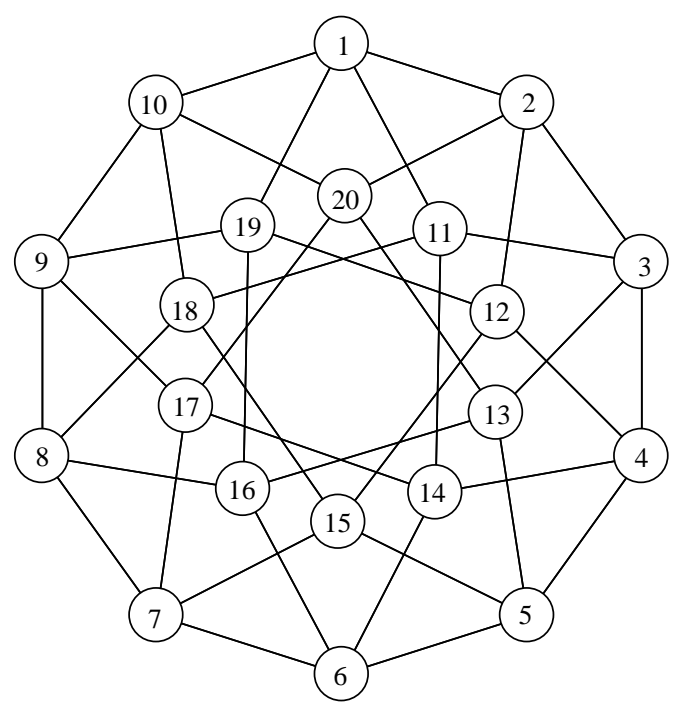

Figure 1: $R_{10}(2,3)$

\section{Symmetry}

The group consisting of all symmetries of $R_{n}(a, r)$ which preserve the cycle of rim edges setwise is dihedral, in fact isomorphic to $D_{n}$. It is generated by the symmetries

$$
\begin{array}{lll}
\rho: & A_{i} \rightarrow A_{i+1}, & B_{i} \rightarrow B_{i+1}, \\
\mu: & A_{i} \rightarrow A_{-i}, & B_{i} \rightarrow B_{-a-i} .
\end{array}
$$

Notice that the symmetry $\rho$ has two orbits of length $n$. This tells us that the graphs $R_{n}(a, r)$ are bicirculant. The classification of edge-transitive bicirculants is a topic of interest to many authors, and classifying edge-transitive $R_{n}$ 's is an important step in that project.

The action of $D_{n}$ on the edges has three orbits: one is the set of rim edges, one is the set of hub edges, and the third consists of all in-spokes and out-spokes. The symmetry $\mu \rho$ reverses the edge $\left\{A_{0}, A_{1}\right\}$, and so if $R_{n}(a, r)$ is edge-transitive, its group must act transitively on darts.

It sometimes happens that there is a symmetry interchanging the rim and hub edges:

Lemma 2. There is a symmetry $\sigma$ sending rim edges to hub edges and vice-versa if and only if $r^{2}= \pm 1$ and $r a= \pm a$.

Proof. If the two equations $r^{2}= \pm 1$ and $r a= \pm a$ hold, then by (2) above, we can assume that $r a=-a$. Then the symmetry $\sigma$ given by $A_{i} \rightarrow B_{r i}, B_{i} \rightarrow A_{r i}$ accomplishes this interchange.

On the other hand, suppose that $\sigma$ is a symmetry which interchanges rim and hub edges. Then the hub edges must form an $n$-cycle and so $(r, n)$ must be 1 . By first applying the correct element of $D_{n}$, we can assume that $A_{0} \sigma=B_{0}$ and that $B_{0} \sigma=A_{0}$. By the correct choice of $r$ as in (2), we can assume that $A_{1} \sigma=B_{r}$. Then it must be that $A_{2} \sigma=B_{2 r}$, etc., and so in general, $A_{i} \sigma=B_{r i}$. Now, $B_{1} \sigma$ is a rim vertex, say $A_{s}$. Then in general, $B_{i} \sigma=A_{s i}$. Then $\sigma^{2}$ sends $A_{0}$ to itself and $A_{1}$ to $A_{r s}$. Thus $r s= \pm 1$. Now consider the spoke edges joining $A_{i}$ to $B_{i}$ to $A_{i+a}$. Then $\sigma$ sends these vertices to $B_{r i}, A_{s i}, B_{r i+r a}$, 
respectively, and so these must be joined by spoke edges. More precisely, one is an in-spoke, the other, an out-spoke Thus, one of these two systems must hold for all $i \in \mathbb{Z}_{n}$ :

(1) $\quad r i=s i \quad r i+r a+a=s i$

(2) $r i+r a=s i \quad r i+a=s i$

Letting $i=1$ in system (1) clearly yields $r a=-a$ and $r^{2}= \pm 1$. In system (2), we conclude the $r a=a$, and so $a=(s-r) i$ for all $\mathrm{i}$. Letting $i=0$ shows that $a=0$, which is not allowed.

We are interested in answering three questions about rose window graphs:

(1) For which $n, a, r$ is $R_{n}(a, r)$ edge-transitive?

(2) When it is edge-transitive, what is the order of its symmetry group? This is equivalent to asking for the size of $H$, the stabilizer of one dart.

(3) For which $n, a, r$ is $R_{n}(a, r)$ the underlying graph of a rotary map?

From our remarks above, we can see that the graph is edge-transitive exactly when it has some symmetry sending a rim edge to some spoke.

In the next sections we will present four families of rose window graphs. For each one we will prove that each member of the family is edge-transitive, we will discuss the order and structure of its group, and consider which of its members occur in rotary maps. We will use the word "occur" throughout this paper to mean "occur as the underlying graph of some rotary map".

At the end we will conjecture that every edge-transitive rose window graph belongs to one of these families and that the maps described include all rotary maps on rose window graphs.

\section{Family (1)}

The first family to consider is all graphs of the form $R_{n}(2,1)$. These graphs are called wreath graphs, $W(n, 2)$, and may also be described as $C_{2 n}(1, n+1)$. See [5] for definitions of these graphs.

Figure 2 shows the case where $n=12$.

In this family and others it is convenient to relabel the hub vertices: let $C_{i}=B_{i-1}$. Then the vertices $A_{i}$ and $C_{i}$ have precisely the same neighbors. A graph which has two such vertices is called unworthy and has a "local" symmetry which interchanges those two vertices, leaving all others fixed. In our example, let $\sigma_{i}$ be the symmetry which interchanges vertices $A_{i}$ and $C_{i}$ while leaving all other vertices fixed. Such a symmetry sends a rim edge to a spoke edge, and so $R_{n}(2,1)$ is edge-transitive.

The collection of pairs $\left\{A_{i}, C_{i}\right\}$ forms a system of imprimitivity for the action of the group on the vertices. The kernel of the action on the blocks is $Z=<\sigma_{0}, \sigma_{1}, \sigma_{2}, \ldots, \sigma_{n-1}>$, which is isomorphic to $\mathbb{Z}_{2}^{n}$. When $n=4$, the graph is isomorphic to $K_{4,4}$. In all other cases, $\operatorname{Aut}\left(R_{n}(2,1)\right)$ is generated by $D_{n}$ and $\sigma_{0}$, and so is isomorphic to $\mathbb{Z}_{2}^{n} \rtimes D_{n}$. As $\sigma_{0}^{\rho^{i}}=\sigma_{i}$, the symmetry group has order $2 n\left(2^{n}\right)$; thus a vertex-stabilizer has order $2^{n}$ and a dart-stabilizer has order $2^{n-2}$.

A cycle in a graph $\Gamma$ is consistent provided there is a symmetry of $\Gamma$ which moves vertices in the cycle one step along it. Such a symmetry is called a shunt for the cycle. The remarkable theorem of Conway and Biggs [1] shows that a dart-transitive action on a graph of degree $d$ has exactly $d-1$ orbits of consistent (directed) cycles. In the graph $R_{n}(2,1)$, these are the 

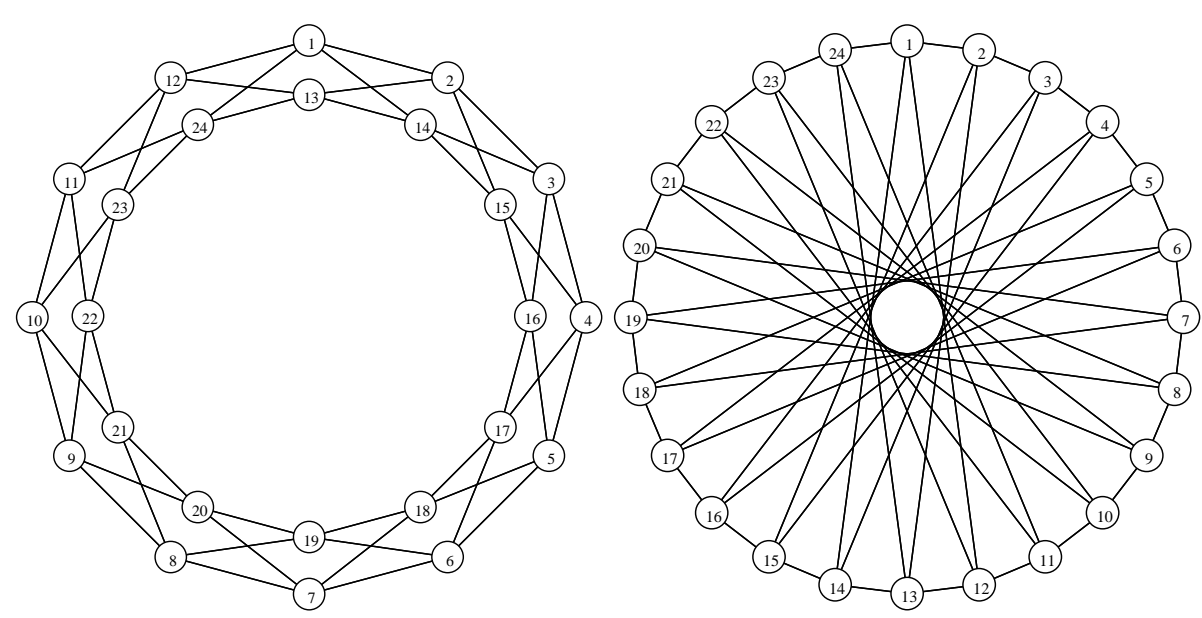

Figure 2: $R_{12}(2,1)=C_{24}(1,13)$

4-cycles, such as $\left[A_{0}, A_{1}, C_{0}, C_{1}\right]$, the $n$-cycles (the rim cycle is one), and the $2 n$-cycles such as $\left[A_{0}, A_{1}, A_{2}, \ldots, A_{n-1}, C_{0}, C_{1}, C_{2}, \ldots, C_{n-1}\right]$.

If $R_{n}(2,1)$ underlies a rotary map $\mathcal{M}$, its faces must be consistent cycles. Since each edge meets only one of the consistent 4 -cycles, the faces must be $n$-cycles or $2 n$-cycles. Then in each face, indices of vertices must occur in the circular order $1,2,3, \ldots$. Without loss of generality, we can assume that the central face is $f=\left[A_{0}, A_{1}, A_{2}, \ldots, A_{n-1}\right]$ and that $\rho$ is the face-rotation $R$, or that $f^{\prime}=\left[A_{0}, A_{1}, A_{2}, \ldots, A_{n-1}, C_{0}, C_{1}, \ldots, C_{n-1}\right]$ is the central face and the shunt $\rho^{\prime}=\rho \sigma_{0}$ is $R$. Notice that $\left(\rho^{\prime}\right)^{n}$ is equal to the product of all $\sigma_{i}^{\prime} s$.

We first consider reflexible maps. Then there must be a symmetry $\beta$ which fixes $A_{0}$ and $A_{1}$, while sending the central face to an adjacent face. If $\mathcal{M}$ is the map and $G=\operatorname{Aut}(\mathcal{M})$ is its symmetry group, then $\beta$ must be in $H=G \cap Z$, and in fact it must be the unique nonidentity element of $H$ fixing $A_{0}$ and $A_{1}$. $H$ must be generated by $\beta$ and all of its conjugates under $D_{n}$.

The group $H$ and the element $\beta$ must satisfy these conditions:

(1) $H$ is invariant under conjugation by $D_{n}$

(2) the orbit of $f$ (resp. $f^{\prime}$ ) under $H$ must be the set of all faces.

(3) $H$ is generated by $\beta$ and its conjugates under $\rho$ and $\mu$.

(4) if $R=\rho$, the number of faces is 8 and so $|H|=8$.

(5) if $R=\rho^{\prime}$, the number of faces is 4 . Then either $|H|=4$ and $\left(\rho^{\prime}\right)^{n} \notin H$ or $|H|=8$ and $\left(\rho^{\prime}\right)^{n} \in H$

(6) no two distinct elements of $H$ agree on any three consecutive indices.

The possibilities for $\beta$ and $H$ satisfying these requirement are limited. To describe them, we introduce this notation: for any divisor $d$ of $n$, and and subset $S$ of $\mathbb{Z}_{d}$, let $\sigma_{d, S}$ be the product of all $\sigma_{i}$ 's such that $i$ is equivalent mod $d$ to some number in $S$. For example, $\sigma_{3,\{2\}}=$ $\sigma_{2} \sigma_{5} \sigma_{8} \ldots \sigma_{n-1}$. Then $\sigma_{d, S} \sigma_{d, T}=\sigma_{d, S \Delta T}$, where $S \Delta T$ is the symmetric difference of the sets $S$ and $T$. Also $\left(\rho^{\prime}\right)^{n}=\sigma_{d, \mathbb{Z}_{d}}$ for any divisor $d$ of $n$. 
It will also simplify our considerations if we regard elements of $H$ as being in $\mathbb{Z}_{2}^{n}$; abbreviate $\sigma_{0}^{a_{0}} \sigma_{1}^{a_{1}} \sigma_{2}^{a_{2}} \ldots, \sigma_{n-1}^{a_{n-1}}$ by the $n$-tuple $\left(a_{0}, a_{1}, a_{2}, \ldots, a_{n-1}\right)$. Then the requirement that $\beta$ fixes $A_{0}$ and $A_{1}$, while sending the central face to an adjacent face says that $\beta=(0,0,1, x, \ldots, z, y, 1)$ for some $x, y, z \in \mathbb{Z}_{2}$. The conjugate of $\beta$ by $\rho \mu$ is then $(0,0,1, y, z \ldots, x, 1)$; as this agrees with $\beta$ in four consecutive places, it must be equal to $\beta$, and so $x=y$.

If $x=y=0$, then $\beta=(0,0,1,0, \ldots, z, 0,1)$, and the conjugate of $\beta$ by $\rho^{3}$ is $(z, 0,1,0,0,1,0, \ldots)$, which again must be equal to $\beta$. Thus, the entries must repeat every 3 places, and so $\beta=\sigma_{3,\{2\}}$. The resulting $H$ is the set of all $\sigma_{3, S}$, and so $\left(\rho^{\prime}\right)^{n}$ is included. Thus, if $n$ is divisible by 3 , we get two reflexible maps from $H$, one with faces of size $n$, one with faces of size $2 n$.

On the other hand, if $x=y=1$, then $\beta=(0,0,1,1, w, \ldots, a, z, 1,1)$, for some $w, a \in$ $\mathbb{Z}_{2}$. Again $z=w$. There are two subcases:

(i) if $z=w=0$, then the conjugate of $\beta$ by $\rho^{4}$ is $(a, 0,1,1,0,0,1,1,0, \ldots)$, which again must be equal to $\beta$. Thus $\beta$ repeats every 4 entries, and so $\beta=\sigma_{4,\{2,3\}}$; here, $H$ is the set of all $\sigma_{4, S}$ such that $|S|$ is even. Again, this group has size 8 and contains $\left(\rho^{\prime}\right)^{n}$, so it works for both sizes of face.

(ii) if $z=w=1$, then $\beta=(0,0,1,1,1, \ldots, a, 1,1,1)$, and its conjugates under $<$ $\rho>$ include $(1,0,0,1,1,1, \ldots, a, 1,1),(1,1,0,0,1,1,1, \ldots, a, 1)$, and,$(1,1,1,0,0,1,1$, $1, \ldots, a)$. The group generated by these four alone has size 16 , and so is eliminated.

This shows that $\sigma_{3,\{2\}}$ and $\sigma_{4,\{2,3\}}$ are the only possibilities for $\beta$ that satisfy the requirements, and so we have found all reflexible maps on graphs in family (1).

We summarize these maps and their properties:

Case I: $n$ is divisible by 3 . Let $n=3 k$, let $\mathcal{M}_{1}$ be the map using $R=\rho$ and $\mathcal{M}_{2}$ be the map using $R=\rho^{\prime}$.

If $k$ is odd, then $\mathcal{M}_{1}=P\left(\mathcal{M}_{2}\right), \mathcal{M}_{1}$ is of type $\{3 k, 4\}_{6 k}$ and is a k-fold covering of the octahedron with k-fold branching points at the face-centers. It follows that $\mathcal{M}_{2}$ is of type $\{6 k, 4\}_{3 k}$ and is a similar k-fold covering of the torus map $\{6,3\}_{2,0}$

If $k$ is even then both maps are self-Petrie. $\mathcal{M}_{1}$ is of type $\{3 k, 4\}_{3 k}$ and is a k-fold covering of a certain map of type $\{6,4\}_{6}$ on the orientable surface of genus $3 . \mathcal{M}_{2}$ is of type $\{6 k, 4\}_{6 k}$ and is a $\frac{k}{2}$-fold covering of the hemi-octahedron, and hence is non-orientable.

Case II: $n$ is divisible by 4 . Let $n=4 k$, let $\mathcal{M}_{3}$ be the map using $R=\rho$ and $\mathcal{M}_{4}$ be the map using $R=\rho^{\prime}$.

Then $\mathcal{M}_{3}$ is self-Petrie of type $\{4 k, 4\}_{4 k}$. It is a k-fold cover of the torus map $\{4,4\}_{2,2}$ (see [2]).

Map $\mathcal{M}_{4}$ is the map $B(4,8 k, 2 k-1,0)$ described in [4]. It is then a k-fold cover of $B(4,8,3,0)$, and so is orientable.

Thus we have found all reflexible maps on family (1) rose window graphs. No chiral maps on these graphs are known to exist and we conjecture that there are none.

\section{Family (2)}

We now consider graphs of the form $\Gamma=R_{2 m}(m+2, m+1)$. For example, Figure 3 shows $R_{12}(8,7)$.

Again, it is convenient to re-label the hub vertices: let $C_{i}=B_{i-1}$, so that the edges are: 


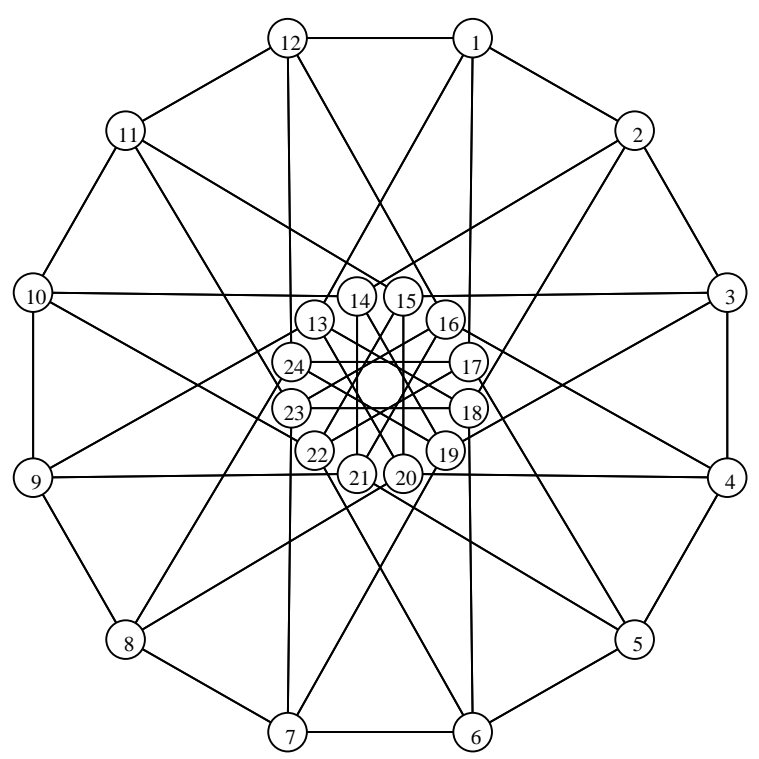

Figure 3: $R_{12}(8,7)$

Rim: $\quad A_{i}-A_{i+1}$

In-Spoke: $\quad A_{i}-C_{i+1}$

Out-spoke: $\quad C_{i}-A_{i+m+1}$

Hub: $\quad C_{i}-C_{i+m+1}$

and then the symmetries $\rho$ and $\mu$ are:

$$
\begin{aligned}
\rho: & A_{i} \rightarrow A_{i+1}, & C_{i} \rightarrow C_{i+1}, \\
\mu: & A_{i} \rightarrow A_{-i}, & C_{i} \rightarrow C_{m-i} .
\end{aligned}
$$

Then we can arrange part of the graph to look like Figure 4:

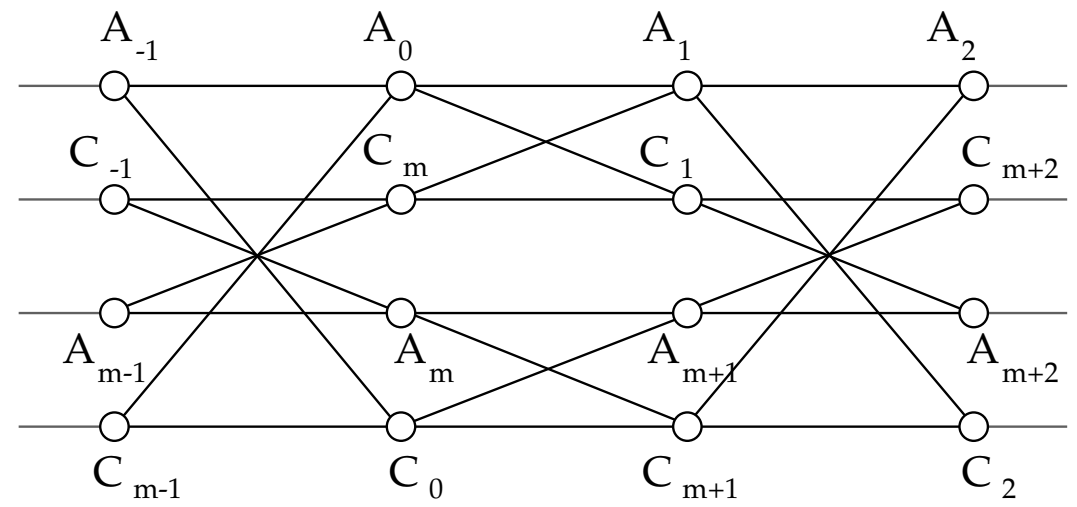

Figure 4: Part of $R_{2 m}(m+2, m+1)$

It is clear that the permutation $\left(A_{0}, C_{0}\right)\left(A_{m}, C_{m}\right)\left(A_{1}, C_{m+1}\right)\left(A_{m+1}, C_{1}\right)$ is a symmetry 
of the graph, and as it sends rim edges to spokes and hubs, the graph must be edge-transitive.

We gather the following from Figure 4: First, if $m>3$, then the graph has girth 4, and if $m>4$, then the only 4 -cycles are those of the form $\left[A_{i}, A_{i+1}, C_{i+m}, C_{i+1}\right]$. If $m>4$, then every edge belongs to exactly one of these cycles. It follows that for $m>4$, the equivalence relation generated by being opposite in a 4-cycle has as its equivalence classes the sets $L_{i}=\left\{A_{i}, A_{i+m}, C_{i}, C_{i+m}\right\}$. Then the collection of all $L_{i}$ is a block system for the action of the symmetry group on the vertices. This last is also true when $m=3$, but not when $m=4$.

For the rest of this general discussion, we will assume that $m>4$; the exceptional cases $m=3,4$ we will discuss at the end.

Let $\sigma_{i}=\left(A_{i} C_{i}\right)\left(A_{i+m}, C_{i+m}\right)\left(A_{i+1} C_{i+m+1}\right)\left(A_{i+m+1} C_{i+1}\right)$; with all other vertices fixed. Then $\sigma_{m+i}=\sigma_{i}$ and any $\sigma_{i}, \sigma_{j}$ commute, and so $Z=<\sigma_{0}, \sigma_{1}, \sigma_{2}, \ldots, \sigma_{m-1}>$ is an elementary abelian 2-group of order $2^{m}$. Clearly, $Z$ is the kernel of the action of $\operatorname{Aut}(\Gamma)$ on the block system $\left\{L_{i}\right\}$. That action is dihedral, isomorphic to $D_{m}$. In fact, if we let $\rho^{\prime}=\rho \sigma_{0}$, then $\rho^{\prime}$ has order $m$ and sends $L_{i}$ to $L_{i+1}$. Moreover $\mu^{\prime}=\mu \rho^{m}$ conjugates $\rho^{\prime}$ to its inverse. Thus $\left\langle\mu^{\prime}, \rho^{\prime}\right\rangle$ is isomorphic to $D_{m}$, and so $\operatorname{Aut}(\Gamma) \cong \mathbb{Z}_{2}^{m} \rtimes D_{m}$.

Now we wish to determine rotary maps on these graphs. The first step is to determine the consistent cycles. As $\rho \mu \sigma_{0}$ is a shunt for $\left[A_{0}, A_{-1}, C_{0}, C_{m-1}\right]$, the 4-cycles are consistent. Clearly $\rho$ is a shunt for the rim cycle $f=\left[A_{0}, A_{1}, A_{2}, \ldots, A_{2 m-1}\right]$, and it is not hard to see that $\rho^{\prime}$ is a shunt for the $m$-cycle $f^{\prime}=\left[A_{1}, A_{2}, \ldots, A_{m-1}, C_{m}\right]$. Thus the lengths of consistent cycles in this graph are $4, m, n$. As in family (1), each edge belongs to only one 4-cycle, and so any rotary map on this graph must have faces of size $m$ or $n$. No chiral maps are known on these graphs, and we conjecture that none exist.

We proceed to construct several families of reflexible maps. If $\mathcal{M}$ is a reflexible map on $\Gamma=R_{2 m}(m+2, m+1)$, let $G=\operatorname{Aut}(\mathcal{M})$. With no loss of generality, we can assume that one face of such a map is $f$ or $f^{\prime}$. The symmetry $\mu$ reverses $f$, leaving $A_{0}$ fixed, while $\mu^{\prime}$ reverses $f^{\prime}$, leaving $C_{m}$ fixed. As the point stabilizers of each of these cycles is trivial, $\mu$ and $\mu^{\prime}$ are the only symmetries reversing those cycles and leaving those vertices fixed. Thus $G$ must contain $\rho$ and $\mu$ as generators of the stabilizer of face $f$ or $\rho^{\prime}$ and $\mu^{\prime}$ as generators of the stabilizer of face $f^{\prime}$.

Any symmetry $\beta$ which acts as a reflection interchanging the two faces on either side of a given edge must be in the kernel of the action of $G$ on the block system $\left\{L_{i}\right\}$, i.e., in the subgroup $H=G \cap Z$, and thus must be a product of $\sigma_{i}$ 's. Such a reflection at the edge $\left\{A_{0}, A_{1}\right\}$, for instance, must fix those two vertices, but move $A_{2}$ and $A_{2 m-1}$ (in $f$ ) or $C_{m-1}$ (in $f^{\prime}$ ). Thus the product must include $\sigma_{-2}$ and $\sigma_{2}$, but neither $\sigma_{-1}, \sigma_{0}$ nor $\sigma_{1}$.

That reflection $\beta$, followed by the face-rotation $\rho$ or $\rho^{\prime}$, is movement one step along a Petrie path, and so the order of $\beta \rho$ or $\beta \rho^{\prime}$ is the length of a Petrie path. As Petrie paths are consistent cycles in a reflexible map, these must also have length $m$ or $n$.

We find it clearest to describe the map by describing the group $H$; the set $F$ of faces of the map, is simply the orbit of $f$ or $f^{\prime}$ under $H$. What are the properties that $H$ must have?

The group $H$ and the element $\beta$ must satisfy these conditions:

(1) $H$ is invariant under conjugation by $D_{m}$

(2) the orbit of $f$ (resp. $f^{\prime}$ ) under $H$ must be the set of all faces.

(3) $H$ is generated by $\beta$ and its conjugates under $\rho$ and $\mu$.

(4) if $R=\rho^{\prime}$, the number of faces is 16 and so $|H|=16$. 
(5) if $R=\rho$, the number of faces is 8. Then either $|H|=8$ and $\rho^{n} \notin H$ or $|H|=16$ and $\rho^{n} \in H$

(6) no two distinct elements of $H$ agree on any four consecutive indices.

As before, we let $\sigma_{d, S}$, where $d$ is a divisor of $m$ and $S$ is a subset of $\mathbb{Z}_{d}$, be the product of all $\sigma_{i}$ such that $i$ is equivalent mod $d$ to some number in $S$.

We consider four groups and the resulting maps:

(1) If $m$ is divisible by 4 , say $m=4 k$, the group of all $\sigma_{4, S}$ certainly has order 16 , contains $\beta=\sigma_{4,\{2\}}$ and $\rho^{m}=\sigma_{4, \mathbb{Z}_{4}}$. Thus it allows maps of face size $m$ and $n$. By using the relation $\sigma_{i} \rho=\rho \sigma_{i+1}$, it is not hard to see that if $k$ is odd, the maps are of types $\{n, 4\}_{m}$ and $\{m, 4\}_{n}$ and are Petrie to each other, while if $k$ is even, they are of types $\{n, 4\}_{n}$ and $\{m, 4\}_{m}$ and are self-Petrie. The maps of types $\{m, 4\}_{n}$ and $\{m, 4\}_{m}$ are $k$-fold covers of the torus map $\{4,4\}_{2,2}$, with $k$-fold branching at the face-centers. Those of type $\{n, 4\}_{n}$ are $2 k$-fold covers of the torus map $\{4,4\}_{4,0}$, with similar branching.

(2) If $m$ is divisible by 5 , say $m=5 k$, let $H$ be the group of all $\sigma_{5, S}$ for sets $S$ of even size. Then $H$ has 16 elements, but does not contain $\rho^{m}=\sigma_{5, \mathbb{Z}_{5}}$. Thus it allows only maps of face size $m$. $H$ contains $\beta=\sigma_{5,\{2,3\}}$, and the order of $\beta \rho^{\prime}$ is $m$, and so this construction gives maps of type $\{5 k, 4\}_{5 k}$ for all $k$. These maps are $k$-fold branched coverings of the map $\{5,4\}_{5}$ having 40 edges. The maps are all non-orientable and self-Petrie.

(3) If $m$ is divisible by 6 , say $m=6 k$, one group that satisfies the requirements is generated by $\beta=\sigma_{6,\{2,3,4\}}$ and all of its conjugates under $\rho$. The sets $S$ for elements in the group are: $012,123,234,345,045,015,03,14,25,0134,0235,1245,024,135,012345$ as well as the null set, 16 in all. Since $\mathbb{Z}_{6}$ is in this list, both $n$ - and $m$-cycles are allowed.

As in construction (1), odd values for $k$ give a Petrie pair of maps of types $\{n, 4\}_{m}$ and $\{m, 4\}_{n}$, while even values of $k$ give two self-Petrie maps of types $\{n, 4\}_{n}$ and $\{m, 4\}_{n}$.

(4) Another group that satisfies (1)-(6) in the case $m=6 \mathrm{k}$ is the set of all $\sigma_{6, S}$ such that $S$ contains an even number of odd numbers and an even number of even numbers. This is the group $H$ generated by $\beta=\sigma_{6,\{2,4\}}$ and its conjugates under $\rho$. $H$ is of order 16 but does not contain $\rho^{m}$, and so will construct maps of types $\{6 k, 4\}_{6 k}$ for all $k$.

It should be noted that different constructions yield nonisomorphic maps. For instance, at $m=24$, the graph $\Gamma=R_{48}(26,25)$ allows three reflexible maps of type $\{24,4\}_{24}$, one each from constructions (1), (3) and (4). These are three distinct, nonisomorphic maps.

We have shown then, that if $m$ is divisible by 4,5 , or 6 , then the graph $R_{2 m}(m+2, m+1)$ has a reflexible embedding in some surface. An argument similar to the one for Family (1) shows that the four groups presented are the only ones satisfying conditions $[1],[2],[3]$ and so, that we have described all rotary maps on Family (2) graphs in the general case.

To handle the exceptional cases, first consider $m=3$. The resulting graph $R_{6}(5,4)$ is isomorphic to the skeleton of the cuboctahedron, i.e., to the medial graph of the cube. Its group is the group of either polyhedron, and so is isomorphic to $\mathbb{Z}_{2}^{3} \rtimes D_{3}$. Then the stabilizer of a vertex in $R_{6}(5,4)$ is the same as the stabilizer of an edge in the cube, and that is isomorphic to $\mathbb{Z}_{2}^{2}$. Thus no rotary map exists on this graph.

Second, we consider $m=4$, the graph $R_{8}(6,5)$. This graph is isomorphic to $Q_{4}$, the skeleton of the 4-dimensional cube. Thus its group is $\mathbb{Z}_{2}^{4} \rtimes S_{4}$, of order $16 \cdot 24=384$. This graph is well-known and occurs in two rotary maps. One is $\mathcal{M}=\{4,4\}_{4,0}$, a reflexible map of type $\{4,4\}_{8}$; the other is its Petrie, of type $\{8,4\}_{4}$.

The paper [3] introduces a family $C(p, r, s)$ of edge-transitive graphs. Family (2) of rose window graphs arise as a special case: $R_{2 m}(m+2, m+1) \cong C(2, m, 2)$. 


\section{Family (3)}

The third family of edge-transitive rose window graphs are those $R_{n}(a, r)$ for which

(1) $n$ is even, so $n=2 m$ for some $m$

(2) $a$ is even, $a=2 b$, such that $b^{2} \equiv \pm 1(\bmod m)$

(3) $r$ is odd and $r \equiv 1(\bmod m)$.

(4) $R_{n}(a, r)$ is in neither family (1) nor family (2).

We separate Family (3) into (3a) in which $b^{2} \equiv 1(\bmod m)$ and $(3 \mathrm{~b})$ in which $b^{2} \equiv-1$ $(\bmod m)$.

Define a permutation $\sigma$ of the vertices as follows:

In family (3a):

$$
A_{i} \sigma=\left\{\begin{array}{ll}
A_{b i} & \text { if } i \text { is even } \\
B_{b i-b} & \text { if } i \text { is odd }
\end{array}, \quad B_{i} \sigma= \begin{cases}A_{1+b i} & \text { if } i \text { is even } \\
B_{b i-b+r} & \text { if } i \text { is odd }\end{cases}\right.
$$

In family (3b):

$$
A_{i} \sigma=\left\{\begin{array}{ll}
A_{b i} & \text { if } i \text { is even } \\
B_{b i-b} & \text { if } i \text { is odd }
\end{array}, \quad B_{i} \sigma= \begin{cases}A_{-1+b i} & \text { if } i \text { is even } \\
B_{b i-b-r} & \text { if } i \text { is odd }\end{cases}\right.
$$

It is easy to check that in each case, this $\sigma$ is a symmetry of the graph. As it sends the rim edge $\left\{A_{0}, A_{1}\right\}$ to the in-spoke $\left\{A_{0}, B_{0}\right\}$, the graph must be edge-transitive.

Moreover, notice that in (3a), it happens that $\sigma$, as well as $\mu$ and $\mu \sigma$, interchange the neighbors of $A_{0}$ in pairs, while in (3b), $\sigma$ permutes them in a cycle of length 4 . It is easy to compute that in (3b), the symmetry $\rho^{\prime}=\mu \rho \sigma$ acts as a shunt for the cycle $\alpha=$ $\left[A_{0}, B_{0}, B_{2-r}, A_{r}, A_{1-r}, B_{r-1}, B_{1}, A_{1}\right]$; if $r=1$, this cycle has length 4 , while if $r=m+1$, it has length 8 .

In order to make definitive statements about maps on graphs in this family, we need information on the size of $H$, the stabilizer of a dart. Computer work suggests the following

Conjecture 3. If $\Gamma$ is $R_{n}(a, r)$ in family (3), then the stabilizer of a dart in Aut $(\Gamma)$ is trivial.

Suppose for a moment that this conjecture holds. From this, we conclude that:

(1) In family (3a), the stabilizer of vertex $A_{0}$ consists of exactly four elements. These must be the identity, $\sigma, \mu$ and $\mu \sigma$, all of order 2 . Thus, no graph in family (3a) occurs.

(2) Every graph in family (3b) occurs as the underlying graph of a chiral map. Its faces are the cycles in the orbit of the cycle $\alpha$ shown above. If $r=1$, this map is of type $\{4,4\}$ on the torus. If $r=m+1$, then the map is of type $\{8,4\}$.

To describe those maps a little more precisely, suppose $B$ and $C$ are natural numbers whose greatest common divisor is 2 . Let $n=\frac{B^{2}+C^{2}}{2}$, let $m=n / 2$, and let $x$ and $y$ be the least Bezout multipliers such that $B x+C y=2$. Let $a=|C x-B y|$. Then the underlying graph of the $\operatorname{map}\{4,4\}_{B, C}$ is $R_{n}(a, 1)$ of family (3); every graph in family (3) having $r=1$ arises in this way.

Each map of type $\{8,4\}$ on $\Gamma$ in family (3b) is a twofold cover of one of the torus maps (though not every torus map yields such a cover). The covering is branched at face-centers only. 


\section{Family (4)}

The fourth and last family of edge-transitive rose window graphs are those of the form $R_{12 m}(9 m+2,3 m+1)$ or $R_{12 m}(3 m+2,9 m+1)$. It will be convenient to think of these as $\Gamma=R_{12 m}(3 d+2,9 d+1)$ where $d$ can be $m$ or $-m \bmod n=12 m$.

First consider the permutation $\sigma$ of the vertices defined by:

$$
A_{i} \sigma=\left\{\begin{array}{ll}
A_{i} & \text { if } i \equiv 0(\bmod 3) \\
B_{i-1} & \text { if } i \equiv 1(\bmod 3) \\
B_{i-a+1} & \text { if } i \equiv 2(\bmod 3)
\end{array}, \quad B_{i} \sigma= \begin{cases}A_{1+i} & \text { if } i \equiv 0(\bmod 3) \\
A_{i+a-1} & \text { if } i \equiv 1(\bmod 3) \\
B_{i+6 d} & \text { if } i \equiv 2(\bmod 3)\end{cases}\right.
$$

It is easy to check that $\sigma$ is an involutary symmetry of $\Gamma$, and as it sends the rim edge $\left\{A_{0}, A_{1}\right\}$ to the in-spoke $\left\{A_{0}, B_{0}\right\}$, we see that all such graphs are edge-transitive.

We consider a special case in which $\Gamma$ has another symmetry. Suppose that $m$ is twice an odd number, and let $b=d+1$. Then $a=3 b-1, r=4-3 b$, and it follows that $3 b^{2} \equiv 3$ and $12 b \equiv 12(\bmod 12 m)$. Using that fact it is possible to show that $\tau$ defined by:

$$
A_{i} \tau=\left\{\begin{array}{ll}
A_{b i} & \text { if } i \equiv 0(\bmod 3) \\
B_{b i-b} & \text { if } i \equiv 1(\bmod 3) \\
A_{b+b i-1} & \text { if } i \equiv 2(\bmod 3)
\end{array}, \quad B_{i} \tau= \begin{cases}A_{1+b i} & \text { if } i \equiv 0(\bmod 3) \\
B_{4+b i-4 b} & \text { if } i \equiv 1(\bmod 3) \\
B_{b+b i-1} & \text { if } i \equiv 2(\bmod 3)\end{cases}\right.
$$

is a symmetry of $\Gamma$. Notice that $\tau$ fixes both $A_{0}$ and $A_{-1}$ while moving both $A_{-2}$ and $A_{1}$.

We then divide family (4) into (4a), in which $n$ is an odd multiple of 12 , family (4b), in which $n$ is an odd multiple of 24, and (4c) in which $n$ is an even multiple of 24. Thus $\tau$ exists only for graphs in family (4b).

Theorem 4. Every graph in family $(4 b)$ is the underlying graph of a reflexible map.

Proof. First consider the symmetries $\tau$ and $\mu$. Each is an involution within the stabilizer of vertex $A_{0}$, and the product $S=\tau \mu$ has order 4 . Thus, the group they generate must be of order 8, isomorphic to $D_{4}$. There are two reflexible maps having this group as the vertexstabilizer. One has $R=\rho$ as its face-rotation. It is not difficult to verify that $R S^{-1}$ has order 2. The other map is the Petrie of this one. Its face rotation is $\tau \rho$, and its face-length is $6 g$ where $g$ is the additive order of $3 b-9 \bmod n$.

Computer work suggests the following:

Conjecture 5. The stabilizer of a dart has order 1 in family (4a), 2 in (4b), 1 in (4c).

If the conjecture is true, then no graph in (4a) or (4c) can underlie a rotary map, because the entire stabilizer of $A_{0}$ consists of the identity, $\mu, \sigma$, and $\mu \sigma$, all of order 2 .

Somewhat scantier evidence suggests:

Conjecture 6. If $n$ is a multiple of 48, then the two graphs in family (4) are isomorphic. 


\section{The "if-cycle" theorems}

We present four theorems, each of which says that if a cycle of a certain kind is in the orbit of the rim cycle, then the graph must fall into a corresponding set of families. In the descriptions, we use the phrase "repeating pattern"; for instance "the cycle has repeating pattern [rim, in-spoke, hub, out-spoke]" means that the cycle is made of some $k$ repetitions of this pattern, thusly: $\left[A_{0}, A_{1}, B_{1}, B_{r+1}, A_{r+a+1}, A_{r+a+2}, B_{r+a+2}, B_{2 r+a+2}, A_{2 r+2 a+2}, A_{2 r+2 a+3}\right.$, $B \ldots]$.

Theorem 7. If the graph $\Gamma=R_{n}(a, r)$ admits a symmetry $\sigma$ under which the image of the rim cycle $\left[A_{0}, A_{1}, A_{2}, \ldots, A_{n-1}\right]$ has repeating pattern [rim, in-spoke, hub, out-spoke], then $\Gamma$ is in family (1) or $n$ is divisible by 8 and $\Gamma$ is in family (2).

Theorem 8. If the graph $\Gamma=R_{n}(a, r)$ admits a symmetry $\sigma$ under which the image of the rim cycle $\left[A_{0}, A_{1}, A_{2}, \ldots, A_{n-1}\right]$ has repeating pattern [in-spoke, out-spoke], then $n$ is even, and $\Gamma$ is in family (1) or $\Gamma$ is in family (3).

Theorem 9. If the graph $\Gamma=R_{n}(a, r)$ admits a symmetry $\sigma$ under which the image of the rim cycle $\left[A_{0}, A_{1}, A_{2}, \ldots, A_{n-1}\right]$ has repeating pattern [in-spoke, hub, out-spoke], then $n$ is divisible by 3 and $\Gamma$ is in family (1) or family (3) or family (4).

Theorem 10. If the graph $\Gamma=R_{n}(a, r)$ admits a symmetry $\sigma$ under which the image of the rim cycle $\left[A_{0}, A_{1}, A_{2}, \ldots, A_{n-1}\right]$ has repeating pattern [out-spoke,rim, in-spoke], then $n$ is divisible by 3 and $\Gamma$ is in family (1) or $n$ is divisible by 12 and $\Gamma$ is in family (2) or $n$ is an odd multiple of 24 and $\Gamma$ is in family (4).

We will prove one of these, Theorem 8. The other theorems are proved with similar methods.

Proof of Theorem 8 . We can without any loss of generality assume that there is a symmetry $\sigma$ such that $\left[A_{0}, A_{1}, A_{2}, \ldots, A_{n-1}\right] \sigma=\left[A_{0}, B_{0}, A_{a}, B_{a}, A_{2 a}, B_{2 a}, \ldots\right]$. The length of this second cycle is twice the additive order of $a \bmod n$, and if this length is to be $n$, we must have $(a, n)=2$. Let $a=2 b, n=2 m$, so that $(b, m)=1$. Then, letting $C_{i}=A_{i} \sigma$ and $D_{i}=B_{i} \sigma$, we have that

$$
C_{2 i}=A_{a i} \text { and } C_{2 i+1}=B_{a i} .
$$

It is perhaps simpler to write:

$$
C_{j}= \begin{cases}A_{b j} & \text { if } j \text { is even } \\ B_{b j-b} & \text { if } j \text { is odd }\end{cases}
$$

Now, of the four neighbors of $A_{0}=C_{0}$, we know that $B_{0}=C_{1}$, while $B_{-a}=C_{-1} ; D_{0}$, which also must be a neighbor of $A_{0}=C_{0}$, must then be $A_{1}$ or $A_{-1}$.

Case 1: $D_{0}=A_{1}$. Then $A_{2}$ must be $C_{a}$, which we know is $A_{b a}$. Thus $b a \equiv 2(\bmod n)$, and so $b^{2} \equiv 1 \bmod m$. Then $C_{a+1}=B_{2}$. The common neighbor of $C_{1}$ and $C_{a+1}$ must be $D_{1}$ and it must be a $B$ vertex, and a neighbor of $B_{0}$. By a correct choice for $r$, we can insist that $D_{1}=B_{r}$. Then $r$ is odd and $B_{2}=B_{2 r}$, so $2 r=2$. Thus either $r$ is 1 or $m$ is even and $r=m+1$. 
Case 2: $D_{0}=A_{-1}$. Then $A_{-2}$ must be $C_{a}$, which we know is $A_{b a}$. Thus $b a \equiv-2(\bmod$ $n$ ), and so $b^{2} \equiv-1 \bmod m$. In a similar fashion, we can choose $r$ so that $2 r=2$, and so $r=1$ or $m+1$.

Thus, in either case, $\Gamma$ is in family (1) or (3).

\section{The Big Conjecture}

Conjecture 11. Every edge-transitive Rose Window graph belongs to one of the four families of graphs introduced in this paper.

This conjecture has been verified for all $n \leq 100$.

\section{References}

[1] N. Biggs, Aspects of symmetry in graphs, Algebraic methods in graph theory, Vol. I, II (Szeged, 1978), pp. 27-35, Colloq. Math. Soc. János Bolyai, 25, North-Holland, Amsterdam-New York, 1981.

[2] H. S. M. Coxeter, W. O. J. Moser, Generators and relations for discrete groups, Fourth edition, Ergebnisse der Mathematik und ihrer Grenzgebiete 14., Springer-Verlag, Berlin-New York, 1980.

[3] C. E. Praeger, M. Y. Xu, A characterization of a class of symmetric graphs of twice prime valency, European J. Combin. 10 (1989), no. 1, 91-102.

[4] S. Wilson, Bicontactual regular maps, Pacific J. Math. 120 (1985), no. 2, 437-451.

[5] S. Wilson, Families of Regular Graphs in Regular Maps, J. Comb. Theory, Ser. B 85 (2002), no. 2, 269-289. 